This is a post-peer-review, pre-copyedit version of an article published in IEEE journal of radio frequency identification. The final authenticated version is available online at:

DOI http://dx.doi.org/10.1109/JRFID.2018.2806738

(C) 2018 IEEE. Personal use of this material is permitted. Permission from IEEE must be obtained for all other uses, in any current or future media, including reprinting/republishing this material for advertising or promotional purposes, creating new collective works, for resale or redistribution to servers or lists, or reuse of any copyrighted component of this work in other works. 


\title{
Interference sources in congested environments and its effects in UHF-RFID systems: a review
}

\author{
Josep-Ignasi Cairó, Jordi Bonache, Member, IEEE, Ferran Paredes, Member, IEEE, and Ferran \\ Martín, Fellow, IEEE
}

\begin{abstract}
In an scenario where RFID readers become increasingly common in hand held devices, the radios are prone to several interference, not only from external radio sources, but also from the plurality of portable devices that may become every time more common. For that reason it is of interest to well understand how these radio interference may be influencing a UHF-RFID transceiver working according to EPCglobal Class-1 Gen-2. In particular in this work it is analyzed the combination of interference coming from the self-radio, from other radio systems such as mobile phone or other RFID reader, and finally combine such effects with the appearance of multiple tag antenna interfering each other. A method based on simulation using tag antenna design, is presented to evaluate inter-tag interference in a variety of cases. For a better understanding analytic examples are presented to compute such interference interactions within the RFID system.
\end{abstract}

Index Terms-UHF-RFID, mobile phone, read-range, ACPR, IIP3, Tag antenna.

\section{INTRODUCTION}

$\mathbf{S}$ SYSTEMS using Radio Frequency Identification (RFID) are becoming very popular and wide spread, as the radio technology improves, system integration into the Radio Frequency Integrated Circuit (RFIC) is increasing, antenna is more compact, the price lowers and the performance improves. For those reasons, the possibility to integrate the radio in mobile or portable devices is feasible, and this opens the possibility for its use in even more different situations and environments [2]. The number of applications where RFID is deployed is increasing, and so the number of reader transceivers and Tag antennas.

Due to the longer read range and small antenna size, the UHF-RFID is more attractive as compared to other RFID systems. The UHF-RFID specifications are defined by the EPCglobal Class-1 Gen-2 [3], which implements the ISO 18000-6C, the ISO 29143 air-interface protocol for mobile RFID interrogators, and ISO $18000-6 \mathrm{~A} / \mathrm{B}$ for operation in direct mode [4]. Details such as spurious emission limitations are determined by the local regulations such as ETSI [5] in Europe and FCC [6] in USA, which are to be considered for the coexistence of devices.

There are studies devoted to explain the causes and effects of interference for RFID. Some very important limitations are environmental caused such as type of materials close to the tags (metal, water) that highly affect local permittivity, and

The authors are with CIMITEC, Departament d' Enginyeria Electrònica, Universitat Autònoma de Barcelona, 08193 Bellaterra, Spain. E-mail: josepignasi.cairo@e-campus.uab.cat.

Manuscript received July 4, 2017; revised. other caused by multiple reflections. Others not related to environment are for example self-leakage, which happens when continuous carrier is sent by the reader while receiving circuit is activated and may desensitize the receiver. Due to its strong influence, some authors [7] are proposing methodologies for its compensation within the own chip. Local oscillator phase noise, is also influencing read range, since it will leak to the receiver passing through the power amplifier and isolator, overshadowing thermal noise component from the receiver. This effect can be minimized [8]. Thanks to the short time difference between RFID transmit and backscattering signal, the range correlation effect can further reduce such unwanted influence. In addition other important causes of range reduction are the ones caused by other radios, in adjacent channels or also propagating strong signals in nearby frequency bands.

From our knowledge, there is no work dealing with a combination of possibilities for interference issues that nowadays RFID readers are prone to. So in this work, several sources of interference that may affect the RFID systems when coexisting with various sort of other radios in the UHF band, influencing in the read range are listed and analyzed in detail. Also analytical expressions for such influences are detailed, introducing new aspects for such evaluation such as the mutual tag antenna influence or new radio sources such as LTE (4G) that are penetrating in the UHF band. All of them are analyzed and some analytic examples are presented for better understanding.

\section{Self-Leakage (Self-Jammer) Noise Generation}

In this first section, a detailed analysis is done on the causes that, several sort of either internal and external interference may cause to the radio system, influencing read range when inventorying surrounding tag antennas.

\section{A. Self-leakage (Self-Jammer) range reduction}

In a RFID system, since the reverse modulated signal that a reader receives back from the tags is very weak [9], and due to the continuous carrier wave that reader needs to transmit to the tag to ensure energy supply, this can be coupled through the same PCB design or via reflections by nearby close objects into the receiver, this can lead to sensitivity problems. The cause is the fact the weak tag modulated information will be received by the reader, while the receiver can be saturated by the before mentioned transmit carrier leakage or strong reflected power. This will impose limitations in demodulating the signal, so it will have strong effect in the reading range. 
Fig. 1 shows in an RFID reader provided by AMS, the path for RF signal and the coupling paths that need to be maintained as short as possible. Size and component orientation need to be carefully considered in addition to GND vias and grounding planes.

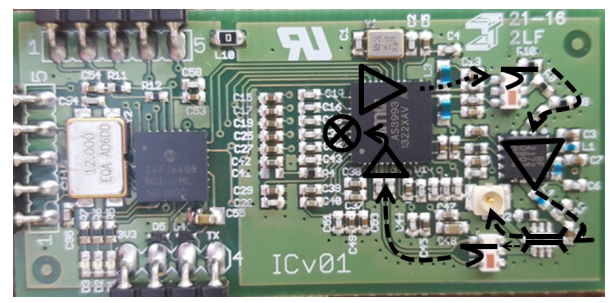

Fig. 1. PCB component distribution for the UHF transmitter and mixer receiver input from a reader based on AS3993 from AMS.

Gen-2 protocol specifies that, when the $T X$ launches command information, the receiver is in non-working state; after the command information is sent, a continuous carrier wave is transmitted and the receiver circuit starts to work simultaneously. For a transmit power of $0.5 \mathrm{~W}(27 \mathrm{dBm})$ and a circulator with $22 \mathrm{~dB}$ isolation (antenna circulator or directional coupler), the carrier leakage will be $5 \mathrm{dBm}$ to the receiver input, which would saturate the receiver first stage $\left(S_{s j}\right.$ in Fig.2). Such receiver desensitizing signal is known as simply carrier leakage, or leaking carrier. The interference produced by the transmitter in the receiver can be one of the big problems in the radio performance, degrading the radio range considerably.

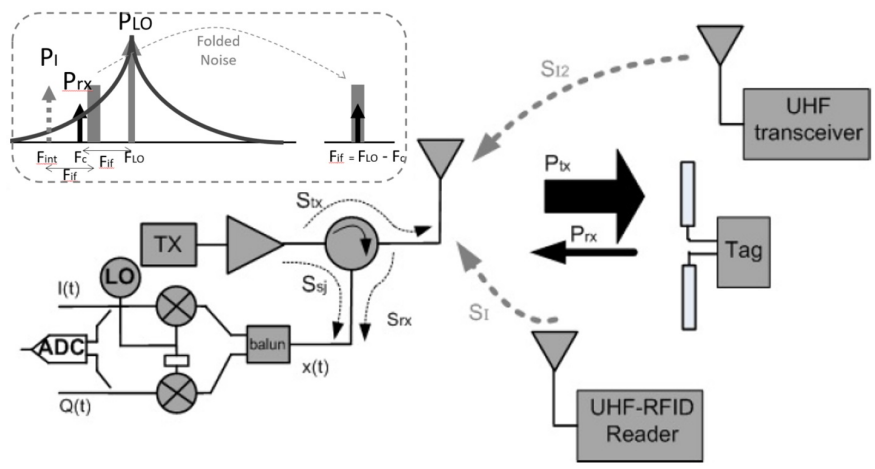

Fig. 2. Interferences caused by other RFID radios in the nearby.

In case the direct current (DC) offset due to TX leakage to the receiving antenna is removed by the baseband Band Pass Filter (BPF), there is also the phase noise of the TX-LO leakage on the receiver bandwidth, that can not be removed by the filter and may have much stronger level than thermal noise present at receiver input, overshadowing the noise in a manner that the reverse link interrogation range mainly depends on the phase noise of the TX leakage (Fig.2).

To evaluate such influence, one must include the nonlinearities effects of the receiver into the self-jammer signal, so the output noise will include such effects when evaluating the signal quality. Let us name $A_{i s o l}$ the isolation between the circulator ports and the output power of the power amplifier (PA) $P_{\text {out }}$ (in our example $22 \mathrm{~dB}$ ). The leakage signal from the output of the PA to mixer input, assuming a receiver with mixer as a first stage instead of typical LNA first stage, is $S_{S J}(t)$ or self-jammer. The third order Input Intercept Point (IIP3) is used to investigate the effects of the receiver nonlinearities into the $S_{S J}(t)$ signal and also an interference signal $S_{I}(t)$ that may be present at the receiver input as well as at an interference frequency $f_{I}$. Describing the output of a receiver as:

$$
y_{m x, \text { out }}(t)=\alpha_{1} x(t)+\alpha_{3} x^{3}(t)
$$

with $\alpha_{1}$ representing the small signal gain and $\alpha_{3}<0$ the nonlinear behavior of the receiver, with $x(t)$ the input signal with desired receive signal $s_{R X}(t)$ and defined as:

$$
\begin{aligned}
x(t)= & s_{R X}(t)+s_{S J}(t)+s_{I}(t)=A_{R X} \cdot \cos \left[\left(\omega_{c} \pm \omega_{R X}\right) t\right] \\
& +A_{S J} \cdot \cos \left(\omega_{c} t+\theta_{S J}\right)+A_{I} \cdot \cos \left[\left(\omega_{c} \pm \omega_{I}\right) t+\theta_{I}\right]
\end{aligned}
$$

it follows that after substituting (2) into (1) and removing the components out of the band, rejected by the internal ICLPF, the minimum amplitude of the output in-band signal at the output of the receiver $y_{m x_{o u t}}$ (when phases $\theta_{S J}=\theta_{I}=$ $0)$, will be depending upon the amplitude signals of the selfjammer $\left(A_{S J}\right)$ and the amplitude of received signal $\left(A_{R X}\right)$, assuming $A_{S J} \gg A_{I}$;

$$
\left|y_{m x_{\text {out }}}\right|=\alpha_{1} A_{R X}\left(1-\frac{9}{4}\left|\frac{\alpha_{3}}{\alpha_{1}}\right| A_{S J}^{2}\right)
$$

So the signal-to-noise ratio at the receiver output is [7]:

$$
\begin{aligned}
S N R_{R X}= & 10 \log \left(\frac{\left|S_{\text {out }}\right|^{2}}{N_{R X}}\right)=10 \log \left(\left(\alpha_{1} A_{R X}\right)^{2}\right) \\
& -N_{R X}(d B)+20 \log \left(1-\frac{9}{4}\left|\frac{\alpha_{3}}{\alpha_{1}}\right| A_{S J}^{2}\right)
\end{aligned}
$$

where $N_{R X}$ is the noise power at the receiver, and the $S_{\text {out }}$ the output signal power. The last term shows the receiver sensitivity degradation, which is directly related to the nonlinearity level of the receiver (represented here with the third level intercept point of the receiver IIP3), which corresponds to:

$$
I I P 3=10 \log \left(\frac{4}{3}\left|\frac{\alpha_{1}}{\alpha_{3}}\right|\right)
$$

The non-linear performance of the mixer as first stage will limit the performance regarding sensitivity degradation. For high IIP3, the radio will be less sensible to self-jammer. When combining expressions (4) and (5), we can obtain that the receiving power including the effect of self-jammer will become:

$$
R_{R X}=S N R+N_{R X}(d B)-20 \log \left(1-\frac{3 \cdot a_{S J}^{2}}{i i p_{3}}\right)
$$

As can be seen in Fig.3, the range degradation is shown for a radio with maximum read range of $14 \mathrm{~m}$, for the case of three different radio specifications of IIP3. For low IIP3 (-10 $\mathrm{dBm}$ ) the degradation in range can be very high even with small self-jammer interference. 


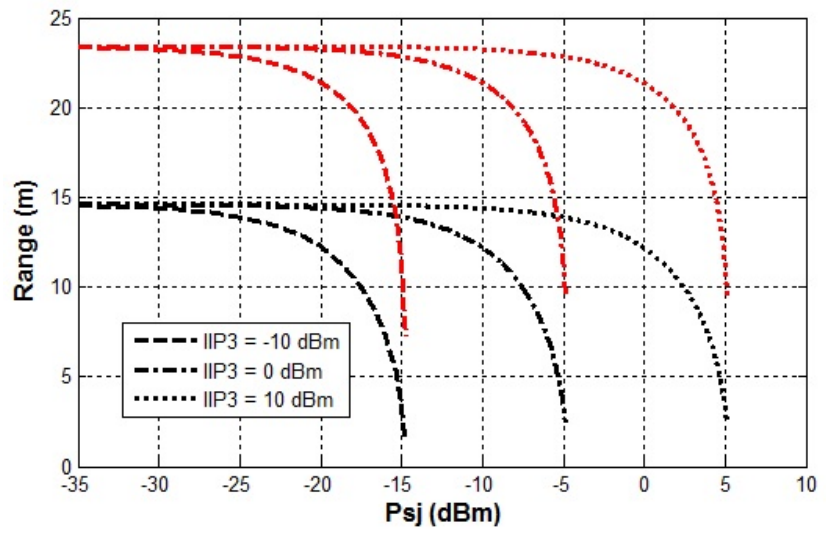

Fig. 3. Read range reduction caused by self-jammer effects, and improved effects caused by Range Correlation (upper curves).

\section{B. Noise power due to phase noise and interferer}

In a dense reader environment, the EPCglobal Gen-2 standard provides for one Adjacent Channel Power Ratio ACPR = $30 \mathrm{dBch}$ and $60 \mathrm{dBch}$ for second adjacent channel. Considering a receiver with a sensitibity of $P_{R X, \min }=-80 \mathrm{dBm}$ and a Channel Bandwidth $\mathrm{CBW}=200 \mathrm{kHz}$ (limited in EU, and 500 $\mathrm{kHz}$ in USA), one can find the phase noise requirements and noise figure of the receiver. We assume a modulation index $m$ between 0.4 and 0.6 the $\mathrm{SNR}=11 \mathrm{~dB}$ for a $\mathrm{BER}=10^{-3}$ and PSK modulation.

1) Phase Noise $\left(\Theta_{L O}\right)$ : Noise power at the receiver, considering the phase noise and interferer is; $P_{N}(d B c / H z)=$ $P_{I}+\Theta_{L O}(\Delta \omega)+10 \log (B W)$. We know also that SNR must comply with the condition of accepting Adjacent Channel Power Rejection Ratio (ACPR) for the $S N R_{m i n}$.

$\frac{P_{R X, \min }}{P_{I} \cdot \Theta_{L O}(\Delta \omega) \cdot C B W} \geq S N R_{\text {min }}$
$\Theta_{L O}(\Delta \omega) \leq P_{R X, \text { min }}+A C P R-S N R_{\text {min }}-10 \log (C B W$

in which case the phase noise needs to be $-115 \mathrm{dBc} / \mathrm{Hz}$.

2) Phase Noise Power $\left(\sigma^{2}\right)$ : Such power will influence the reading range. Evaluation of the reading range will depend on data rate that, in accordance with FM0 modulation EPC Global standard can be between $r_{\max }=640 \mathrm{kbps}$ and $r_{\min }=40 \mathrm{kbps}$, to comply with $B E R=10^{-3}$. If reader transmit power is $P_{T X_{r d}}=+30 \mathrm{dBm}$, and isolation between transmitter and receiver is $A_{\text {isol }}=22 \mathrm{~dB}$ [12].

1) A typical phase noise power spectral density from [10] is $-115 \mathrm{dBc} / \mathrm{Hz}$ relative to the $\mathrm{CW}$ signal power at the offset of the subcarrier frequency of $640 \mathrm{kHz}$. So relative to the $\mathrm{CW}$ signal we have total LO phase noise power at 640 kbps: $N_{\Theta_{L O}}=\Theta_{L O}+10 \log [R($ bps $)]=-115+$ $10 \cdot \log \left(640 \cdot 10^{3}\right)=-56 \mathrm{dBc}$.

2) The phase noise power of the input leaking component at the receiver will be for a transmit power $P_{T X}=30$ $\mathrm{dBm}: N_{\text {leak }}=P_{T X}-A_{\text {isol }}+N_{\Theta_{L O}}=30 \mathrm{dBm}-$ $22 d B-56 d B c=-48 d B m$.

3) With a conversion factor of $C_{p a}=50 \mathrm{~dB}$ from phase to amplitude phase noise, the equivalent phase noise at the receiver will be: $\sigma^{2}=N_{\text {leak }}+C_{p a}=-48 d B m-$ $50 d B=-98 d B m$.

In previous section similar phase noise has been also used, so one can consider the possibility to use similar results as the ones shown in Fig.3. In table I we can see different values for the phase noise when having the interference signals at different frequency locations for a typical $A_{i s o l}+C_{p a}=65$.

TABLE I

PHASE NOISE AT THE RECEIVER

\begin{tabular}{ccc}
\hline \hline $\mathrm{ACPR}(\mathrm{dB})$ & $\Theta_{L O}(\mathrm{dBc} / \mathrm{Hz})$ & $\sigma^{2}(\mathrm{dBm})$ \\
\hline 20 & -124 & -101 \\
30 & -115 & -98 \\
50 & -94 & -71 \\
60 & -84 & -61 \\
\hline
\end{tabular}

\section{Reader range improvement due to the range correlation on phase noise}

In an ideal receiver, the noise floor is caused by thermal noise only. However due to the non perfect isolation between TX and RX antennas we can consider the primary performance limiting on the uplink, the local oscillator (LO) phase noise $\left(\Theta_{L O}\right)$. LO leaking from transmitter to receiver, overshadows the thermal noise component at the receiver input, that is the leaking power $\left(P_{\text {leak }}=P_{T X}-A_{\text {isol }}\right)$ a fundamental limitation in terms of reader sensitivity. One can express the local oscillator signal with its phase noise as:

$$
x_{L O}(t)=A_{L O} \cdot \cos \left[\omega t+\Theta_{L O}(t)\right]
$$

where $A_{L O}$ is the amplitude of the oscillator. The LO signal amplified by the PA and passed through the circulator to the receiver, is transmitted to the air, and is also being received later as backscattered from the tag. The backscattered signal $x_{M}(t)$ in the PSK modulation at the receiving antenna can be expressed as:

$$
x_{M}(t)=\cos \left[\omega\left(t-\frac{2 r}{c}\right)+\Theta_{s}(t)+\Theta_{L O}\left(t-\frac{2 r}{c}\right)\right]
$$

Where $r$ is the tag-reader distance, and $c$ is the wave propagation velocity, $\Theta_{s}(t)$ denotes the phase signal representing the binary data ("0" or "1") of the tag. At the receiver the backsattering signal and the LO signal are mixed, and the output is low-pass filtered. The resulting in-phase baseband signal will be:

$$
x(t)=\cos \left[\Theta_{0}+\frac{2 r}{c}+\Theta_{s}(t)+\triangle \Theta(t)\right]+n_{0}(t)+n_{P N}(t)
$$

where $\Theta_{0}+\frac{2 r}{c}$ is the constant phase shift dependent on the tag-reader distance, $\triangle \Theta(t)$ is the residual phase noise, $n_{0}(t)$ is the thermal noise at the receiver, and $n_{P N}(t)$ is the sum of additive phase noise of the receiver, that do not affect the tag's signal phase which contains information data, so they are not considered. The residual phase term in (10) is given by 


$$
\triangle \Theta(t)=\Theta_{L O}(t)-\Theta_{L O}\left(t-\frac{2 r}{c}\right)
$$

When having the same LO for TX and RX, the phase noise of the received signal is correlated with the LO, depending on the time difference between the two signals. For small time difference (RFID case), the effect greatly abbreviates the phase noise spectrum at baseband, effect known as range correlation [8]. A quantitative characterization of the relation between range and phase noise can be done in the frequency domain. The PSD of $\triangle \Theta(t), S_{\triangle \Theta}\left(f_{0}\right)$ at an offset frequency, $f_{0}$ is given by:

$$
\begin{array}{r}
S_{\triangle \Theta}\left(f_{0}\right)=N_{\Theta_{L O}}\left(f_{0}\right) \cdot \mid 1-e^{-\left.j 2 \pi f_{0} \frac{2 r}{c}\right|^{2}=} \\
N_{\Theta_{L O}}\left(f_{0}\right) \cdot\left[4 \sin ^{2}\left(2 \pi \frac{r f_{0}}{c}\right)\right]
\end{array}
$$

So for a low offset frequency, the baseband noise spectrum will increase proportional to the square of the distance between tag and reader. The correlation level between the phase noise of the received tag signal with that of the LO is inversely proportional to the time difference between the two signals (very small $\sim$ nsec, for the case of RFID due to short distances), and so the phase noise is reduced by correlation effect. For the case of having values for $r$ and $f_{0}$ of $5 \mathrm{~m}$ and $160 \mathrm{kHz}$, respectively, the value of $r f_{0} / c$ will be on the order of $10^{-3}$. So the range correlation effect will reduce the PSD dramatically $\left(\sigma_{R C}^{2}=-128 \mathrm{dBm}\right)$. The improved results in the reading range can be obtained by considering Table I results computation and Fig.3.

\section{RANGE REDUCTION CAUSED BY OTHER RADIOS}

Affecting the read range in RFID systems are also the influence caused by other radios in close proximity, working at nearby frequency bands or adjacent channels. It is very common to share a communication channel with several radio systems that are interconnected aiming at inter-changing information among them in a non-deterministic manner. So there are time intervals that the radios broadcast to the medium in order to interrogate for a possible presence of other radios, in the same band or a band close to our transmitting-band [13]. There may be the case where, a radio transmitting higher power level, may interfere with the receiving radio so they can incur in:

- Presence of other readers that are requested to transmit high power, due to low tag sensitivity and may interfere with our radio.

- De-sensitivity, due to the presence of strong signal from other radio systems (phone, Short Range Devices (SRD), Wireless Sensor Networks (WSN), etc, $\cdots$ ) at the receiver that causes the receiver first stage to saturate and operate wrongly.

\section{A. Mobile phones as interference source}

In order to expand the number of frequencies available to mobile operators, the World Radiocommunications Conference (WRC-07) allocated in 2007 in Region 1 the 790$862 \mathrm{MHz}$ frequency band to mobile services. This new band referred to as Digital Divident band, while allowing the advanced cellular systems that use high speed broadband LTE, there is a concern on potential interference to low power SRD operating in adjacent band 863-870 $\mathrm{MHz}$ [15].

Considering mobile phones a source of interference, there exist two mobile phone bands that are close to RFID-UHF system, such as 800 Band, that in the case of Spain, is becoming the 20 band (B20) for 4G LTE table II (this band has disappeared from TDT channels) also in use in other European countries, and the 900 Band, being used by 3G GSM (3g rural areas, corresponds to B8). Other bands are being used in other countries such as LTE band 5 at $850 \mathrm{MHz}$, used in Corea and Israel and LTE Band 6 of $800 \mathrm{MHz}$ being used in LTE Japan. Due to the proximity to the UHF bands used for the SRD such as our RFID system, there may exist some effects of interference, that could degrade the behavior of the radio, mostly in the upper adjacent band, because of high level of Out-Of-Band (OOB) emissions produced by very wide bandwidth LTE signals Fig.4.

TABLE II

FREQUENCIES AND LTE BANDS

\begin{tabular}{ccccc}
\hline $\begin{array}{c}\text { Frequency } \\
(\mathrm{MHz})\end{array}$ & LTE Band & $\begin{array}{c}\text { Uplink } \\
\text { Freq. }\end{array}$ & $\begin{array}{c}\text { Downlink } \\
\text { Freq. }\end{array}$ & $\begin{array}{c}\text { RFID } \\
(\text { EU) }\end{array}$ \\
\hline 800 & 20 & $832-862$ & $791-821$ & $865-868$ \\
\hline
\end{tabular}

Fig.4 shows in wide dark how the output spectrum mask of a UE transmitter is divided on three components: occupied or channel bandwidth (with $99 \%$ of the total integrated mean power), out of band (OOB) and the out of spurious emission domain [19]. The figure shows the in-band and out-of-band power. A possible case for interference evaluation would be transmitting maximum power $(23 \mathrm{dBm})$ in 16QAM, on all 50 resource blocks within a $10 \mathrm{MHz}$ channel centered on 857 $\mathrm{MHz}$ [11].

With the LTE uppmost $10 \mathrm{MHz}$ channel (852-862 MHz) radio as interfere, from the spectrum mask it is obtained interference power of $P_{I}=-23 \mathrm{dBm}(\mathrm{RBW}=100 \mathrm{kHz})$ at the RFID band. For a SNR of $11 \mathrm{~dB}$ in the RFID $\left(B E R=10^{-3}\right)$, we can obtain from Friis equation:

$$
\frac{C}{I+N}=\frac{S_{r d}}{P_{I} G_{r d}}\left(\frac{\lambda}{4 \pi r}\right)^{2}
$$

In this case for the RFID radio with $-80 \mathrm{dBm}$ sensitivity, and reader antenna gain of $6 \mathrm{dBi}$, the distance where the LTE radio should be located in order not to interfere with the RFID system is minimum $62 \mathrm{~m}$ (considering direct line-of-sight). So for LTE (4G) radios closer than $62 \mathrm{~m}$ from the RFID reader, the effect would be noticeable in tag reading degradation.

In the work done by [14], there are some test performed introducing a RFID system at $868 \mathrm{MHz}$ which is interfered by a GSM phone working at $880.2 \mathrm{MHz}$. The GSM radio located at $1 \mathrm{~m}$ from the RFID reader transmits at $2 \mathrm{~W}$, and changes its transmit power from $33 \mathrm{dBm}(2 \mathrm{~W})$ maximum level down to $5 \mathrm{dBm}$. Table III shows how the radiated field by the GSM signal reduces the reading range. 


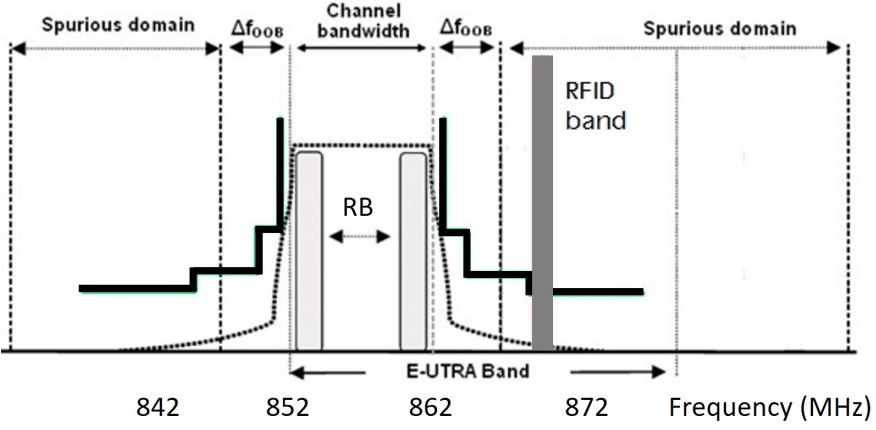

Fig. 4. LTE spectrum mask and example of radiation power.

TABLE III

READ RANGE REDUCTION DUE TO GSM RADIO

\begin{tabular}{ccccccc}
\hline \hline GSM power $(\mathrm{dBm})$ & 5 & 9 & 15 & 21 & 27 & 33 \\
\hline Read Range $(\mathrm{cm})$ & 92 & 62 & 31 & 18 & 11 & 3 \\
\hline
\end{tabular}

\section{B. Interference between readers}

In a scenario where the number of RFID readers starts to increase, it can exist a situation where their interaction and mutual interference may become a real problem. In such cases, it is necessary to investigate the influence they may have on the back-scattering from the tag.

In the case of RFID readers close to each other, there is the possibility of interference when a reader transmits a command signal that interferes with the tag reception of another reader. Thus signals transmitted from distant readers may be sufficiently strong to impede accurate decoding of the back-scattered signals from adjacent tags. Let us imagine a reader transmitting $1 \mathrm{~W}$ output power with antenna gain 6 $\mathrm{dBi}$ and a reader located at a distance " $\mathrm{r}$ " receiving in a $1 \mathrm{dBi}$ antenna (out of main lobe). In case both are tuned to the same channel, the power received at $5 \mathrm{~m}$ distance would be $P_{R X}=$ $-3.5 \mathrm{dBm}$, enough to block the receiver.

For the situation of being in a multiple reader environment, using the EPC Global Gen.2 dense interrogation mode, with the reader transmitting in the adjacent channel, it would transmit with $1 \%$ of the total energy of the principal channel ( $20 \mathrm{~dB}$ down), so at $10 \mathrm{~m}$ distance, the received power by the reader at central channel will be; $P_{R X}^{I}=-9.5 \mathrm{dBm}-20 \mathrm{~dB}=$ $-29.5 \mathrm{dBm}$, sufficient power such as also to block tags at 1 or $2 \mathrm{~m}$ distance.

\section{RANGE REDUCTION CAUSED BY NEARBY TAGS}

One of the largest disadvantages in RFID is the low tag identification efficiency due to multiple tags being in the proximity of the reader. Two causes are identified, one being the fact the multiple tags placed in a shared radiansphere will absorb individually less radiated power than would do one single tag. Another is the tag collision because various tags occupy the same interrogation area and channel. In this event, packets may have to be transmitted and retransmitted until eventually they are correctly received. Since passive tags cannot detect collisions it is necessary a kind of anticollision protocol that enables the recognition of tags with some collisions, but since frame sizes in the protocol are limited, the framed slotted ALOHA algorithm is limited, and also when the number of tags is large, the number of slots required to read tags increases exponentially as the number of tags does. Some methods are proposed, such as [20], in order to try solving such problem, but always including complexity into the system.

A deeper analysis on tag mutual effects is proposed in this analysis to better understand the physical causes for tag reduction in backscattering signal, so reducing reading range. It is considered here the case of having several tag antennas close to each other, and evaluate their mutual effects, in terms of power influence. Electromagnetic coupling will affect each other antennas modifying backscattering radiation pattern, so the matching between antennas and active parts will be influenced and the radiation pattern from the antennas will be modified. In real situations such as retail, large number of tag antennas may be present in close proximity when many items or sensors are to be tagged, (i.e. shopping basket). In such circumstances collective scattering modulation in certain type of tags may lead to confusion or failures in anti-collision procedure in the protocol.

From the optimal design of power antenna transfer in the tag, with matching conditions between the antenna and the tag IC, there can be a deviation from the optimal impedance design, so a mismatch will cause a decrease in sensitivity and read range. When dipole antennas are close, mutual impedance arises at each antenna. In multiple arrayed tags there is also antenna interference that causes a reduction in the radar cross section [21-23]. To experimentally visualize such effect, one can take measurements by examining the two port S-parameters matrix $S$ presenting the power wave reflection coefficient $S$ with the associated power reflection coefficient $|S|^{2}$ Fig.5:

$$
\boldsymbol{S}=\frac{Z_{L}-Z_{a}^{*}}{Z_{L}+Z_{a}} \quad c=\left|\frac{Z_{L}-Z_{a}^{*}}{Z_{L}+Z_{a}}\right|^{2}
$$

with $Z_{L}$ as the load being seen by the antenna, $Z_{a}$ the antenna impedance and, $|S|^{2}$ represents the fraction of the maximum power available from the generator that is not delivered into the load. For an easy interpretation of mutual impedance effects between the antennas, it is recommended converting to $\boldsymbol{Z}$ parameters, where the mutual effects between tag antennas will be represented by the measurement of the mutual components $Z_{12}$ and $Z_{21}$, and the equivalent antenna impedance. Considering mutual effects from other antennas, the equivalent antenna impedance of a tag when the two tags are close is expressed as [24]:

$Z$ in $=50\left[Z_{11}-\frac{Z_{12} Z_{21}}{Z_{22}+Z_{I C}}\right]=50\left[\frac{Z_{11}^{2}+Z_{11} Z_{I C}-Z_{12}^{2}}{Z_{11}+Z_{I C}}\right]$

For the two tags with similar geometry and characteristics $Z$ will be symmetrical (that is to say, $Z_{11}=Z_{22}, Z_{12}=Z_{21}$ ). In this case $Z_{I C}$ is the impedance presented by the Tag IC that should correspond to $Z_{L}$. 


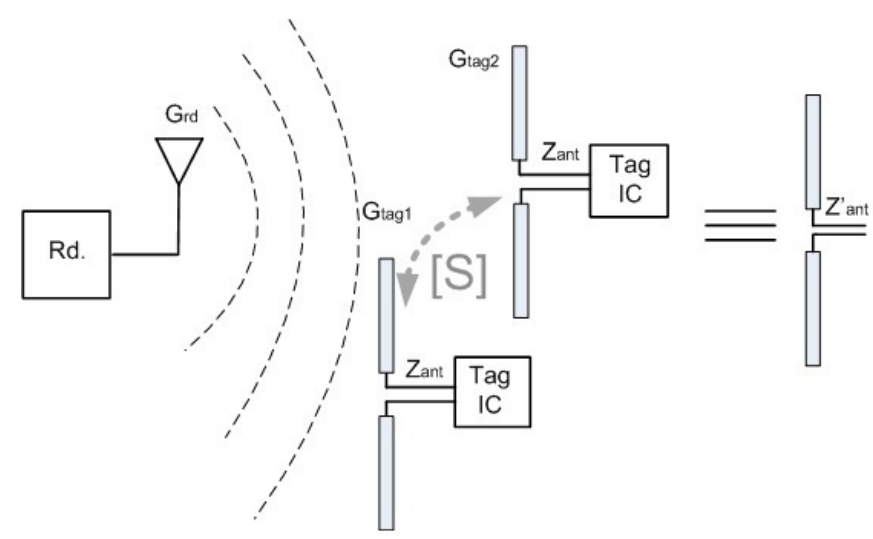

Fig. 5. Effects on reader range of close proximity tags. Also shown RFIDUHF tag design.

To show this effect, a tag dipole antenna has been designed using an electromagnetic 2.5D solver from Agilent (Momentum). It matches an Impinj Monza $5 \mathrm{IC}\left(C_{p}=0.825 \mathrm{pF}, R_{p}\right.$ $=1.8 \mathrm{k} \Omega$ and $C_{i n}=0.245 \mathrm{pF}$ ).

The effects on the influence on mutual tags, has been analyzed based on close proximity in the horizontal and vertical plane axis (Fig.6). In such cases looking at the impedance presented at the tag antenna terminals, there is a clear decrease of the impedance by placing both tags together, so at the extreme of $5 \mathrm{~mm}$ distance, the impedance shift is from the original $Z_{i}=16+j 133$, down to $Z_{f}=9.6+j 9$.
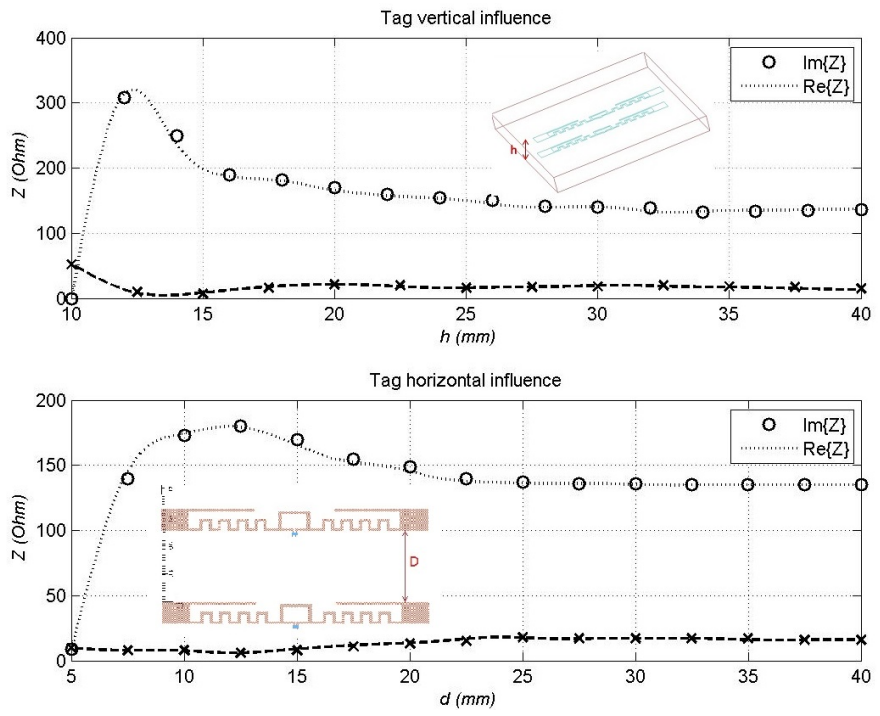

Fig. 6. Simulation results for the influence over tag impedance for the horizontal and vertical close proximity of tags.

The tags influence can be understood as a shadowing effect on the read range. One effect that could be represented by including a factor into the Friis equation, such as a Gain Penalty $\left(G_{p}\right)$ :

$$
P_{R_{t a g}}=P_{T_{r d}} G_{r d}\left(\frac{\lambda}{4 \pi d}\right)^{2} G_{t a g} L_{s y s} G_{P}
$$

here $L_{\text {sys }}$ are the losses in the system and $G_{r d}$ and $G_{t a g}$ are the reader and tag antenna gains. On the other hand, such

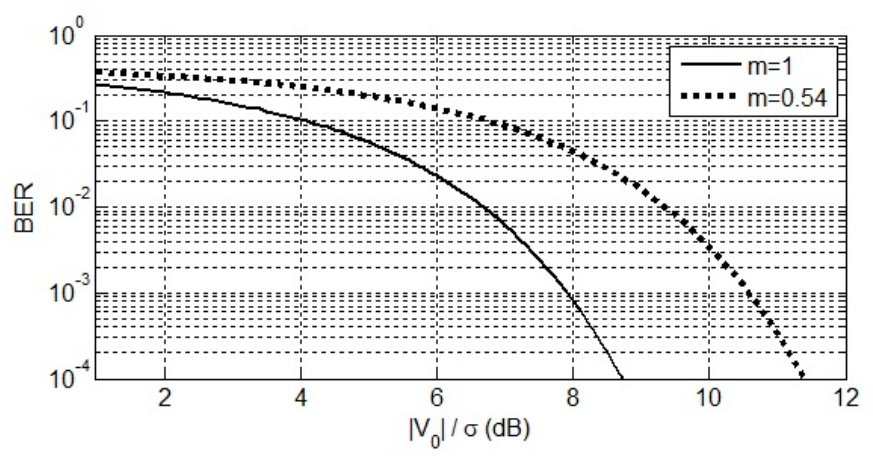

Fig. 7. BER degradation considering two tags close vertically $20 \mathrm{~mm}$.

gain penalty factor, can be analyzed evaluating the influence that such mismatch produced by proximity tags, would have on the voltage induced in the tag. Considering the modulation of the incident electromagnetic field in the antenna, by the tag-IC, such effect is presented in the back-scattering signal affected by nearby tags.

The signal to noise ratio at the receiver is proportional to the energy of the bit so, to the distance between bits in the constellation diagram. According to $d_{\min }=2 V_{0}$, will determine the minimum detection threshold to resolve between all the received bits. Assuming ideal, matched-filter demodulation and Additive White Gaussian Noise (AWGN) with standard deviation $\sigma$ at the detector input, the BER for both PSK and ASK (OOK) will be determined based on such energy distance between received bits. The effect on the backscattering of the tag antennas, can be then introduced by the reflection coefficient between load and antenna impedance affected by proximity tags.

The proximity tags affects the impedance values from the different modulation states, $Z_{1}=R_{1}+j X_{1}$ and $Z_{2}=$ $R_{2}+j X_{2}$ to $Z_{1}$ ' and $Z_{2}$ '. Since for both ASK and PSK, it is possible to express the BER depending on the modulation index $(m)$ as; $m=\frac{\left|\rho_{1}-\rho_{2}\right|}{2}$, being $\rho_{1,2}$ the reflection coefficient at the tag antenna terminals $[25,26]$, it will be possible to include such proximity tag effects into the BER expression.

$$
\begin{aligned}
& B E R=\frac{1}{2} \operatorname{erfc}\left(\frac{\left|V_{R 1}-V_{R 2}\right|}{4 \sqrt{2} \sigma}\right)= \\
& \frac{1}{2} \operatorname{erfc}\left(\frac{\left|V_{0}\right| \cdot\left|\rho_{1}-\rho_{2}\right|}{4 \sqrt{2} \sigma}\right)=\frac{1}{2} \operatorname{erfc}\left(\frac{\left|V_{0}\right| \cdot m}{2 \sqrt{2} \sigma}\right)
\end{aligned}
$$

In ASK modulation, the two possible states, will depend on the modulation depth, which will be conditioned also by the mismatch between antenna and modulation impedances; $\left|V_{R 1}-V_{R 2}\right|=\left|V_{0}\right| \cdot\left|\rho_{1}-\rho_{2}\right|=\left|V_{0}\right| 2 m$. Taking as example the case where two tags are located at a distance of 20 $\mathrm{mm}$ one on top of the other, the simulation results from the mutual coupling, shows that $m=0.54$. The results of BER degradation due to imperfect matching between antennas shows a degradation of SNR of $3 \mathrm{~dB}$, due to only this effect (Fig.7). Such modulation effect, will degrade reading range. 


\section{CONCLUSION}

This paper has addressed several important issues to be considered in early stages when designing real RFID systems, mainly related to the high density concentration of wireless radio sources in the environment. The main sources of interferences for RFID have been explained in detail in order to describe some of the major causes that may degrade the read range of RFID readers. In conclusion, such effects must be taken into consideration at early stages of the design phase in order to minimize them and provide high performance RFID systems.

\section{REFERENCES}

[1] A. E. Abdulhadi and R. Abhari, "Multi-Port UHF RFID tag antenna for enhanced energy harvesting of selfpowered wireless sensor", IEEE Transactions on industrial informatics, vol.12, pp.801-809, Apr.2016.

[2] Z. Rashid , E. Peig and R. Pour, "Bringing Online Shopping Experience to Offline Retail through Augmented Reality and RFID", 5th Int'l Conf. on the Internet of Things (IoT) pp.45-51, 26-28 Oct.2015.

[3] "EPC Radio-Frequency Identification Protocols Calss-1 Generation-2 UHF RFID Protocol for Communications at $860 \mathrm{MHz}-960 \mathrm{MHz}$, Version 1.2.0”, 3rd ed. EPCglobal Inc., Oct.2008.

[4] "ISO-IECCD 18000-6C ver2.1c2", Jul.2005.

[5] "Electromagnetic compatibility and Radio spectrum Matters (ERM); Radio Frequency Identification Equipment operating in the band $865 \mathrm{MHz}$ to $868 \mathrm{MHz}$ with power levels up to $2 \mathrm{~W}$; Part 1: Technical requirements and methods of measurement", ETSI EN 302 208: (V1.1.2).

[6] "Operation Within the Bands 902-928 MHz, 2435-2465 $\mathrm{MHz}, 5785-5815 \mathrm{MHz}, 10500-10550 \mathrm{MHz}$, and 2407524175 MHz", FCC Title 47, pt. 15.

[7] A. Ghahremani, V.D. Razaei, and M.S. Bakhtiar, "A UHF-RFID transceiver with a Blocker-Canceller Feedback and + $30 \mathrm{dBm}$ Output Power", IEEE Transactions on Circuits and Systems - I: Regular Papers, , vol.60, pp.3043-3054, Nov.2013.

[8] A. Droitcour, O. Boric-Lubecke, V. Lubecke, J. Lin, and G.T.A. Kovac "Range correlation and i/q performance benefits in single-chip silicon doppler radars for noncoherent cardiopulmonary monitoring", IEEE Transactions on Microwave Theory Techniques, vol.52, pp.838848, Mar.2004.

[9] Yen, C. Chuan, A. E. Gutierrez and D. Veeramani "Radar cross-section analysis of backscattering RFID tags", IEEE Antennas and Wireless Propagation Letters, vol.6, pp.279-281, Jun.2007.

[10] D. M. Dobkin "The RF in RFID. Passive UHF RFID in Practice", Elsevier, 2008.

[11] Ofcom "LTE User Equipment coexistance with 862 870 MHz", Research document, 2012.

[12] R. Chakraborty, S. Roy and V. Jandhyala "Revisiting RFID link budgets for technology scaling: Range maximization of RFID tags", IEEE Transactions on Microwave Theory Tech. vol.59, pp.496-503, Feb.2011.
[13] S. Pourbagheri, M.S. Bakhtiar, and M. Atarodi "Cellular design for a dense RFID reader environment", IEEE Asia Pacific Conference on Circuits and Systems (APCCAS), pp.1124-1127, Nov.-Dec.2008.

[14] D. Arnaud-Cormos, T. Letertre, A. Diet, and A. Azoulay, "Electromagnetic Environment of RFID Systems", IEEE Proceedings of the 37th European Microwave Conference (EuMA), pp.1652-1655, Oct.2007.

[15] "M66 05R1 SE24 LTE unwanted emissions vs. adjacent 863-870 MHz band", M66 CEPT ECC SE24 meeting documents, 2012.

[16] "AS3993 UHF Single Chip Reader EPC Class 1 Gen2 Compatible", AS3993 Datasheet, AMS, EN v1.

[17] S. Woo, and H. Kim "An Empirical Interference Modeling for Link Reliability Assessment in Wireless Networks", IEEE Transactions on networking, vol.21, pp.272-285, Feb.2013.

[18] D. Gokhale, S. Sen, K. Chebrolu, and B. Raman "On the feasibility of the link abstraction in (rural) mesh networks", IEEE Proceedings in FOCOM, pp.61-65, 13-18 Apr.2008.

[19] "LTE; Evolved Universal Terrestrial Radio Access (EUTRA); User Equipment (UE) radio transmission and reception”, ETSI TS 136 101, V10.7.0, 2012.

[20] F. Zheng and T. Kaiser "Adaptive Aloha anti-collision algorithms for RFID systems", EURASIP Journal on Embedded Sustems Apr.2016.

[21] P.V. Nikitin, K.V.S. Rao, S. F. Lam, V. Pillai, R. Martinez, and H. Heinrich "Power reflection coefficient analysis for complex impedances in RFID tag design", IEEE Transactions on Microwave Theory Techniques, vol.53, pp.2721-2725, Sep.2005.

[22] Pavel, V. Nikitin and K. V. S. Rao, "Effect of Gen2 Protocol Parameters on RFID Tag Performance", IEEE International Conference on RFID, pp.117-122, 27-28 Apr.2009.

[23] Y. Tanaka, Y. Umeda, O. Takyu, M. Nakayama, and K. Kodama, "Change of Read Range for UHF Passive RFID Tags in Close Proximity", IEEE International Conference on RFID, pp.338-345, 27-28 Apr.2009.

[24] H. Yohima, Y. Tanaka, Y. Umeda and O. Takyu, "Analysis of read range for UHF passive RFID tags in close proximity with dynamic impendance measurement of tag ICs", IEEE Radio and Wireless Symposium (RWS), pp.110-113, 16-19 Jan.2001.

[25] U. Karthaus and M. Fisher, "Fully integrated passive UHF RFID transponder IC with $16.7 \mathrm{uW}$ minimum RF input power", IEEE Journal of Solid-State Circuits, vol.38, pp.1602-1608, Oct.2003.

[26] F. Fuschini, C. Piersanti, F. Paolazzi and G. Falciasecca, "On the efficiency of Load Modulation in RFID Systems operating in real environment", IEEE Antennas and Wireless Propagation Letters, , vol.7, pp.243-246, Mar.2008. 


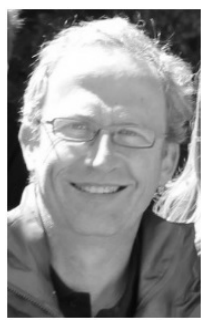

Ignasi Cair was born in Terrassa (Barcelona), Spain in 1967. He received the electronics and telecommunication degrees by Politechnical Universisty of Catalonia, Barcelona, Spain in 1988 and 1992 respectively. He joined the research and development department from the group Circutor in 1995, where he was involved in industrial power electronics and RFID systems. From 1999 until 2003 he joined Philips Semiconductors. In Nijmegen, the Netherlands he was developing GSM power amplifiers using BiCMOS process and later in San Jose, California, engaged in the developments of first integrated WLAN transceiver awarded by 2001 price. From 2003 he has been with Epson Electronics GmbH in Barcelona, leading the R\&D Lab. in RF projects such as antennas, CMOS device characterization, transceiver and system design, from narrow band to ultra-wide band. At 2010 he joined IREC Institute for Energy Research of Catalonia), as senior researcher and project leader devoted to energy efficiency, microgrids and smart grid technologies, communications and renewable integration activities. Currently involved in wireless communications and RFID systems. He holds 27 patents and a long list of publications in conferences and relevant journals.

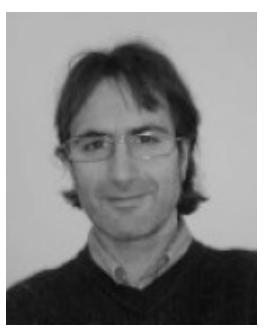

Jordi Bonache (S05M07) was born in Barcelona, Spain, in 1976. He received the physics and electronics engineering degrees and Ph.D. degree in electronics engineering from the Universitat Autnoma de Barcelona (UAB), Barcelona, in 1999, 2001, and 2007, respectively. In 2000, he joined the High Energy Physics Institute, Barcelona, where he was involved with the design and implementation of the control and monitoring system of the MAGIC telescope. In 2001, he joined the Department of Electronics Engineering, UAB, where he is currently an Associate Professor. From 2006 to 2009, he was an Executive Manager with CIMITEC, Drachten, The Netherlands, where he currently leads the research in RFID and antennas. His current research interests include active and passive microwave devices, metamaterials, antennas, and RFID.

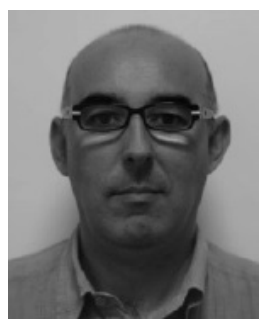

Ferran Martin (M'04-SM'08-F'12) was born in Barakaldo, Spain, in 1965. He received the B.S. degree in physics and Ph.D. degree from the Universitat Autnoma de Barcelona (UAB), Barcelona, Spain, in 1988 and 1992, respectively. From 1994 to 2006 he was Associate Professor in Electronics at the Departament dEnginyeria Electrnica (Universitat Autnoma de Barcelona), and since 2007 he is Full Professor of Electronics. In recent years, he has been involved in different research activities including modelling and simulation of electron devices for high frequency applications, millimeter wave and $\mathrm{THz}$ generation systems, and the application of electromagnetic bandgaps to microwave and millimeter wave circuits. He is now very active in the field of metamaterials and their application to the miniaturization and optimization of microwave circuits and antennas. He is the head of the Microwave Engineering, Metamaterials and Antennas Group (GEMMA Group) at UAB, and director of CIMITEC, a research Center on Metamaterials supported by TECNIO (Generalitat de Catalunya). He has organized several international events related to metamaterials, including Workshops at the IEEE International Microwave Symposium (years 2005 and 2007) and European Microwave Conference (2009), and the Fifth International Congress on Advanced Electromagnetic Materials in Microwaves and Optics (Metamaterials 2011), where he has acted as chair of the Local Organizing Committee. He has acted as Guest Editor for three Special Issues on Metamaterials in three International Journals. He has authored and co-authored over 500 technical conference, letter, journal papers and book chapters, he is co-author of the book on Metamaterials entitled Metamaterials with Negative Parameters: Theory, Design and Microwave Applications (John Wiley \& Sons Inc. 2008), author of the book Artificial Transmission Lines for RF and Microwave Applications (John Wiley \& Sons Inc. 2015), and he has generated 16 PhDs. Ferran Martn has filed several patents on metamaterials and has headed several Development Contracts. Prof. Martn is a member of the IEEE Microwave Theory and Techniques Society (IEEE MTT-S). He is reviewer of the IEEE Transactions on Microwave Theory and Techniques and IEEE Microwave and Wireless Components Letters, among many other journals, and he serves as member of the Editorial Board of IET Microwaves, Antennas and Propagation and International Journal of RF and Microwave Computer-Aided Engineering. He is also a member of the Technical Committees of the European Microwave Conference (EuMC) and International Congress on Advanced Electromagnetic Materials in Microwaves and Optics (Metamaterials). Among his distinctions, Ferran Martn has received the 2006 Duran Farell Prize for Technological Research, he holds the Parc de Recerca UAB Santander Technology Transfer Chair, and he has been the recipient of two ICREA ACADEMIA Awards (calls 2008 and 2013). He is Fellow of the IEEE since 2012 and Fellow of the IET since 2016.

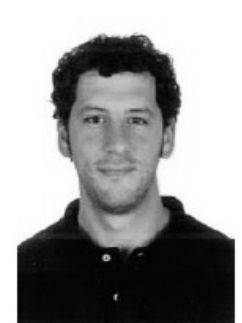

Ferran Paredes was born in Badalona (Barcelona), Spain in 1983. He received the Telecommunications Engineering Diploma (specializing in Electronics) and the Telecommunications Engineering degree from the Universitat Autnoma de Barcelona in 2004 and 2006, respectively and the $\mathrm{PhD}$ degree in Electronics Engineering from the same university in 2012. He was Assistant Professor from 2006 to 2008 at the Universitat Autnoma de Barcelona, where he is currently working as a Research Assistant. His research interests include metamaterial concepts, passive microwaves devices, antennas and RFID. 